\title{
Article \\ Efficient Wireless Monitoring and Control of a Grid-Connected Photovoltaic System
}

\author{
Jonathan Medina-García ${ }^{1}$, Aránzazu D. Martín ${ }^{2, * \mathbb{D}}$, Juan M. Cano ${ }^{2}$, Juan A. Gómez-Galán ${ }^{1}$ (D) \\ and Adoración Hermoso ${ }^{1}$ \\ 1 Department of Electronic Engineering, Computer Systems and Automatics, University of Huelva, \\ 21007 Huelva, Spain; jonathan.medina@diesia.uhu.es (J.M.-G.); jgalan@diesia.uhu.es (J.A.G.-G.); \\ hermoso@diesia.uhu.es (A.H.) \\ 2 Department of Electrical Engineering, University of Huelva, 21007 Huelva, Spain; juan.diaz@die.uhu.es \\ * Correspondence: aranzazu.delgado@die.uhu.es
}

Citation: Medina-García, J.; Martín, A.D.; Cano, J.M.; Gómez-Galán, J.A.; Hermoso, A. Efficient Wireless Monitoring and Control of a Grid-Connected Photovoltaic System. Appl. Sci. 2021, 11, 2287. https:// doi.org/10.3390/app11052287

Academic Editor: Alvaro Araujo Pinto

Received: 6 February 2021

Accepted: 1 March 2021

Published: 4 March 2021

Publisher's Note: MDPI stays neutral with regard to jurisdictional claims in published maps and institutional affiliations.

Copyright: (C) 2021 by the authors. Licensee MDPI, Basel, Switzerland. This article is an open access article distributed under the terms and conditions of the Creative Commons Attribution (CC BY) license (https:// creativecommons.org/licenses/by/ $4.0 /)$.

\begin{abstract}
The design, monitoring, and control of photovoltaic (PV) systems are complex tasks that are often handled together, and they are made even more difficult by introducing features such as real-time, sensor-based operation, wireless communication, and multiple sensor nodes. This paper proposes an integrated approach to handle these tasks, in order to achieve a system efficient in tracking the maximum power and injecting the energy from the PV modules to the grid in the correct way. Control is performed by means of an adaptive Lyapunov maximum power point tracking (MPPT) algorithm for the DC/DC converters and a proportional integral (PI) control for the inverters, which are applied to the system using low latency wireless technology. The system solution exploits a low-cost wireless multi-sensor architecture installed in each DC/DC converter and in each inverter and equipped with voltage, current, irradiance, and temperature sensors. A host node provides effective control, management, and coordination of two relatively independent wireless sensor systems. Experimental validation shows that the controllers ensure maximum power transfer to the grid, injecting low harmonic distortion current, thus guaranteeing the robustness and stability of the system. The results verified that the MPPT efficiency is over $99 \%$, even under perturbations and using wireless communication. Moreover, the converters' efficiency remains high, i.e., for the DC/DC converter a mean value of $95.5 \%$ and for the inverter $93.3 \%$.
\end{abstract}

Keywords: photovoltaic monitoring; smart grids; MPPT; low latency; wireless communication

\section{Introduction}

There are two essential reasons why the use of renewable energy is attractiveenvironmental protection and the increasing demand for energy consumption. Among the different renewable energy sources, solar energy through photovoltaic systems (PV) is rapidly spreading in recent years as solar farms or distributed photovoltaic systems. As it is widely known, solar energy has several advantages such as cleanness, broad availability, and low maintenance, but it has a limited energy conversion efficiency, which makes it crucial to guarantee the correct operation of the PV system components. That refers to the whole PV system, consisting of the PV modules connected in series and/or parallel, and the DC/DC and DC/AC converters when they are connected to the power grid. Moreover, improving system performance in PV installations requires monitoring, which makes the continuous investigation of advanced monitoring and control techniques a necessity to keep up with the advances of the technology. Monitoring focuses on measuring production and checking the operation of the converter and communication devices. The monitoring and control systems for PV installations include sensors, a data acquisition system, and data analysis algorithms [1].

The PV modules are responsible for converting photon energy into electrical power, and they are exposed to outdoor environments, hence to the negative effects of soiling, 
heavy rain, hail, strong wind, etc. To maintain their efficiency close to the nominal efficiency, it is required to monitor their main parameters with sensors to detect if the solar modules suffer any fault, partial shading conditions, or aging degradation. With the appearance of these conditions, the hot spots [2], in the PV modules can make the PV system efficiency decrease and accelerate the degradation of the modules. Therefore, the sensors to detect early faults play an important role in PV systems.

On the other hand, the DC/DC converter transforms the voltage from one DC level to another DC level. In this case, the DC/DC converter used is a boost topology, which can only increase the input voltage level. The control of the boost converter is responsible for searching the maximum power point (MPP) through an MPPT algorithm. There is a wide variety of MPPTs, from the simplest to the most complex, and all of them have the same goal-searching for the MPP. Usually, the MPPT algorithms obtain the voltage that provides the MPP and the system works at that voltage, controlling the DC/DC converter duty cycle. The most used MPPT is the perturb and observe (P\&O) algorithm [3], which is very simple and easy to implement. Other simple and linear MPPT algorithms are the incremental conductance [3], or the ripple correlation control [4]. When there are perturbations, the response of the linear systems can be slower or commit the stability of the system. Then, other MPPT algorithms are used, such as the sliding mode control [5], the fuzzy logic [6], neural networks [7], particle swarm optimization [8], etc. [9]. The non-linear backstepping control is another algorithm used as MPPT [10]. The controller provides the reference voltage to be achieved to work at the MPP by means of the regulation of the duty cycle. This type of control is robust and guarantees global stability. In this study, the adaptive Lyapunov control is used to control the boost converter. The adaptive Lyapunov control is similar to the backstepping control, the difference being that the former takes into consideration the degradation of the components of the DC/DC converter, such as the inductor and the capacitor, to make the system work more efficiently and robustly.

Then, the DC/AC converter is connected at the output of the DC/DC converter when the PV system is grid-connected. The inverter is responsible for converting the DC voltage into AC voltage. Moreover, the DC/AC converter injects the energy from the PV system into the power grid. To provide the energy from the PV modules to the grid in the correct way, a zero-crossing detector [11] is required to inject the voltage in phase with the grid voltage and guarantee the grid stability [12] and power quality [13]. These problems arise in distributed PV systems when a large number of DC/AC converters are connected. The reason for the instability of these kinds of systems is the intermittent nature of solar energy since it depends on environmental conditions. That may cause fluctuations in the grid and over-voltage, making the voltage regulation difficult. Different control strategies have been proposed to reduce the reactive power [14], stabilize the voltage [15], or achieve maximum energy transfer [16]. In this study, the DC/AC converter uses a proportional integral (PI) control to supply energy to the grid. This method is simple and obtains a proper system operation [17].

The integration of the distributed PV systems in the power grid entails technological improvements in the power systems, such as updated sensors and communication, in order to detect the faults in the solar modules [18], control the DC/DC and DC/AC converter to search for and transfer the maximum power, and guarantee the correct operation of the whole system, ensuring the power quality, the stability of the power grid, and a safe operation and maintenance [19].

Wireless sensor networks (WSN) have become popular in the last decade and represent an opportunity in photovoltaic applications as opposed to wired ones, given their different constraints in terms of design and resources. For WSNs, resource constraints refer to the limited amount of energy, communication range, bandwidth, or limitations in processing power and data storage. The design is constrained by the environment to be monitored and is application-specific. Nevertheless, there are clear advantages that wireless technologies offer. The lack of cabling reduces costs and improves scalability and flexibility. They are easy to deploy, reliable, and require lower maintenance than the wired solutions. 
The components required for the deployment of WSNs have seen improvements in costs, size, and power constraints, mainly due to recent advances in microelectronics and wireless technologies. However, the widespread deployment of WSNs requires addressing a variety of challenges, which have to be researched. In this study, collecting precise real-time information on photovoltaic system performance is essential to track efficiently the MPP and guarantee the reliability of the power grid. Therefore, several design aspects must be adequately addressed, such as low latency for a suitable performance of the controllers, robustness against interference, reliability in the wireless data transmission, accuracy in data acquisition, and synchronization between sensor nodes and coordinator nodes. Although ZigBee and Bluetooth protocols have been used in PV systems for the monitoring of currents, voltages, temperature, and irradiance, [20-25], providing energyefficient designs, they cannot comply with tight latency and reliability requirements. This fact has required the design of a tailored wireless sensor network for the target application based on low latency techniques to achieve real-time monitoring and stable performance of the controllers. The Institute of Electrical and Electronics Engineers (IEEE) 802.15.4 standard in beacon-enabled mode and with a guaranteed time slot (GTS) mechanism has been used to meet the data monitoring with a predetermined latency.

For a better understanding of the readers, a list of symbols along with their descriptions that are used in this article are given in Table 1.

Table 1. List of symbols with their descriptions.

\begin{tabular}{cc}
\hline Symbols & Description \\
\hline MPP & maximum power point \\
WPPT & maximum power point tracking \\
WSN & wireless sensor networks \\
WCPVS & wireless grid-connected PV system \\
WSPS & wireless centralized control \\
WCU & wireless smart photovoltaic system \\
CCM & wireless conversion unit \\
GTS & continuous conduction mode \\
BO & guaranteed time slot \\
SO & Beacon Order \\
\end{tabular}

\section{System Architecture Overview}

Figure 1 shows the two-stage, grid-connected wireless PV system. It consists of PV modules connected to a DC/DC converter that transfers the energy to the grid through a DC/AC converter. It has two main building blocks-a wireless grid-connected PV system (WGPVS) that links with a wireless centralized control system (WCC). More specifically, the WGPVS is composed of several wireless smart photovoltaic systems (WSPS) connected to a wireless conversion unit (WCU). Then, there are different WGPVSs connected to the AC bus to transfer all the energy provided by the PV modules to the power grid. The WCC includes the coordinator node that controls all the WGPVSs. Both DC/DC and DC/AC converters are connected to a wireless sensor network where data collected by motes are periodically transmitted to the coordinator node through wireless communication. Then, two control strategies are applied in parallel to generate the required signals for the converters, optimizing the energy conversion of the overall system.

The WSPS includes PV arrays connected to boost converters and wireless sensor network nodes. The boost converter is responsible for managing the MPP of each PV array. For this purpose, the boost converter is connected to the sensor node, which gathers the signals of the required parameters and sends them to the coordinator node. Then, the WCC provides to the sensor node the control signal for the DC/DC converter. Finally, each $\mathrm{DC} / \mathrm{DC}$ converter is connected to the DC bus to transfer the energy. 


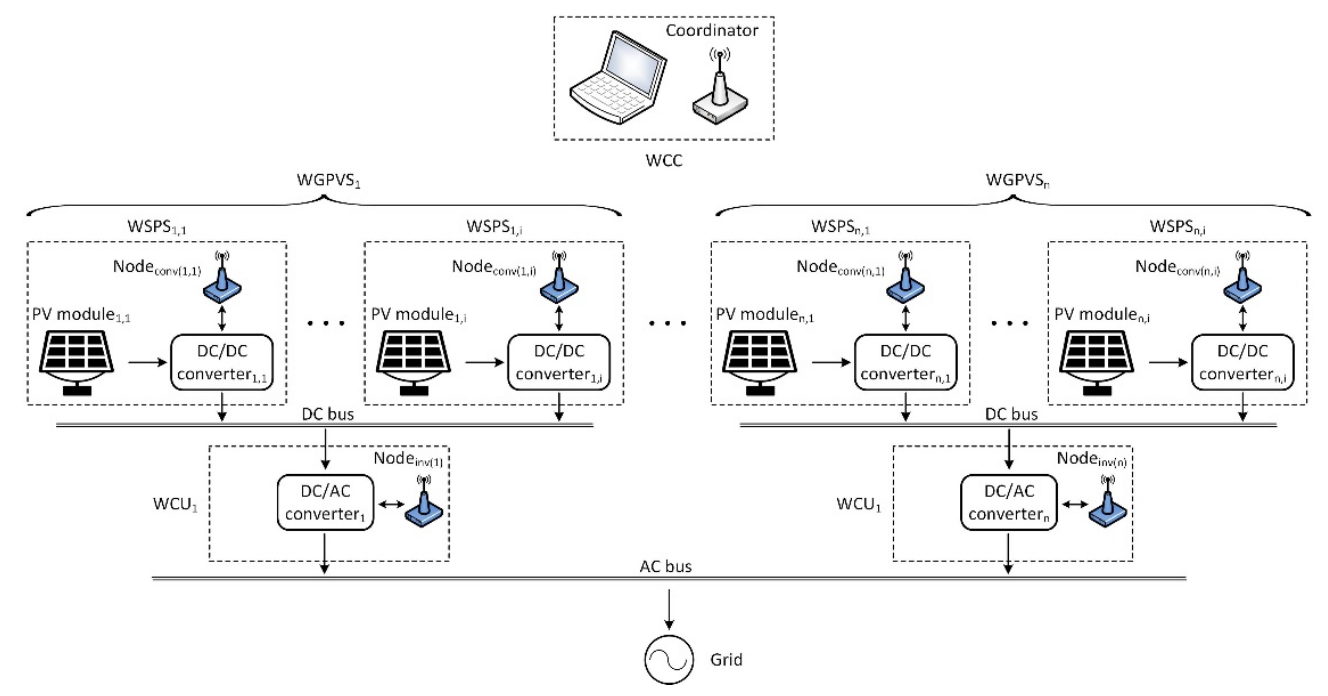

Figure 1. Architecture of the two-stage wireless grid-connected photovoltaic (PV) system.

The $\mathrm{DC} / \mathrm{AC}$ converter is also connected to the $\mathrm{DC}$ bus to transfer the energy from the WSPS to the AC bus and then to the power grid, and convert the DC into AC. This part is called WCU and it is composed of the DC/AC converter connected to a wireless sensor network node, which is responsible for providing the required pulse-width modulation (PWM) signal to inject the energy generated by the PV modules into the grid. For that, the sensor node collects the required values of the inverter and then sends them to the coordinator node. In this case, the control strategy in the WCC is a PI that generates the control signal, which is provided to the inverter also by the sensor node. Moreover, a zero-crossing detector is required to synchronize the signal generated by the DC/AC converter with the grid. Thus, the inverter injects energy into the AC bus.

Finally, the WCC consists of the wireless centralized network node, known as host or coordinator node. The host node is responsible for linking the wireless nodes of the DC/DC converter and the DC/AC converter by means of the IEEE 802.15.4 standard. In addition, the host also has to manage the communication in the sensor network and sends the generated control signals. Thus, the WCC contains the calculations of the control signal for the DC/DC converter to search for the MPP depending on the sensor measurements and the control strategy. The controller applied in this study is the adaptive Lyapunov control for the boost converter and the PI control for the inverter to obtain the control signals to inject the energy into the grid and to synchronize the voltages.

\section{Description and Design of the Photovoltaic System}

\subsection{Wireless Smart Photovoltaic System (WSPS)}

\subsubsection{PV Modules}

In this research, each PV module provides a maximum power of $20.1 \mathrm{~W}_{\mathrm{p}}$ for the standard conditions, with a maximum power voltage of $17.5 \mathrm{~V}$ and a maximum power current of $1.15 \mathrm{~A}$. The open-circuit voltage is $21.6 \mathrm{~V}$ and the short-circuit current is $1.28 \mathrm{~A}$. The commercial PV modules used are the MS-20P (Sumsol, Madrid, Spain). In this case, the experiments have been conducted for three PV modules series-connected.

In order to know the values of the MPP for different environmental conditions, the current-voltage (I-V) and power-voltage (P-V) characteristic curves of the PV module are obtained in simulation and in experimental tests, as Figure 2 depicts. For example, for $910 \mathrm{~W} / \mathrm{m}^{2}$ and $42{ }^{\circ} \mathrm{C}$, the MPP is $19.8 \mathrm{~W}$ for a voltage of $17 \mathrm{~V}$. 

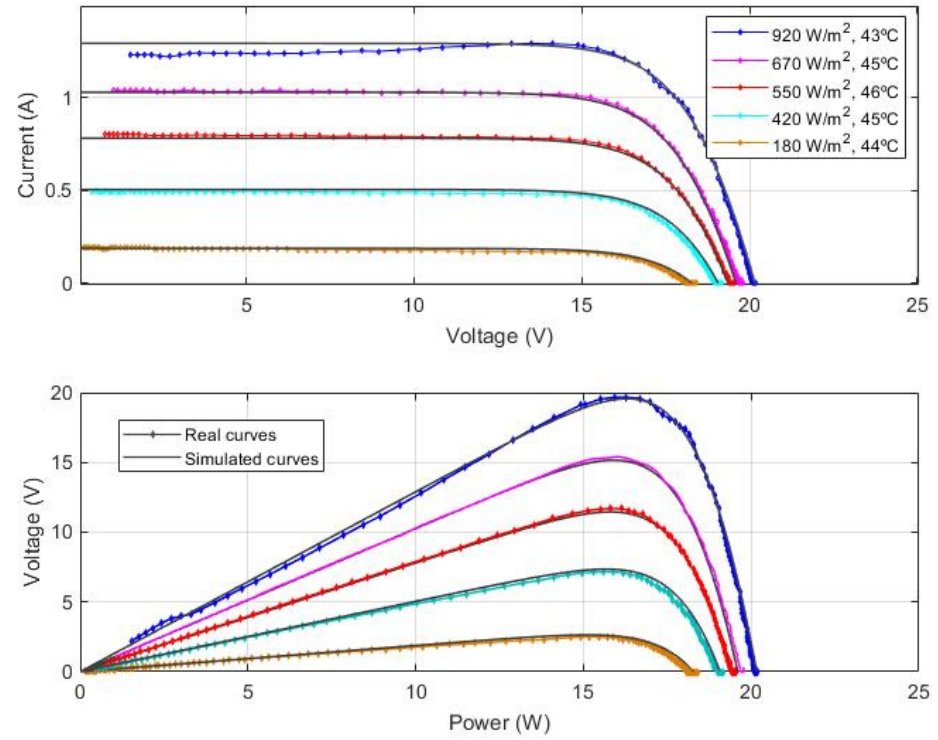

Figure 2. I-V and P-V curves in simulation and experimental tests.

\subsubsection{Boost DC/DC Converter}

The DC/DC converter used in the PV system is a boost converter. This device, responsible for power transmission, steps up the input voltage of the solar array. The boost converter is composed of an input capacitor with a value of $1000 \mu \mathrm{F}$, an inductor of $20 \mathrm{mH}$, a CSD19536KCS MOSFET (Texas Instruments, Dallas, TX, USA) driven by an IR2112 (Ariat Technology Limited in Kowloon, Hong Kong, China), an MBR10200 (Taiwan Semiconductor, Mouser España) diode, and an output capacitor, $C_{0}$, with a value of $4700 \mu \mathrm{F}$. The connection of the converter is shown in Figure 3, in which the electrical circuit is presented.

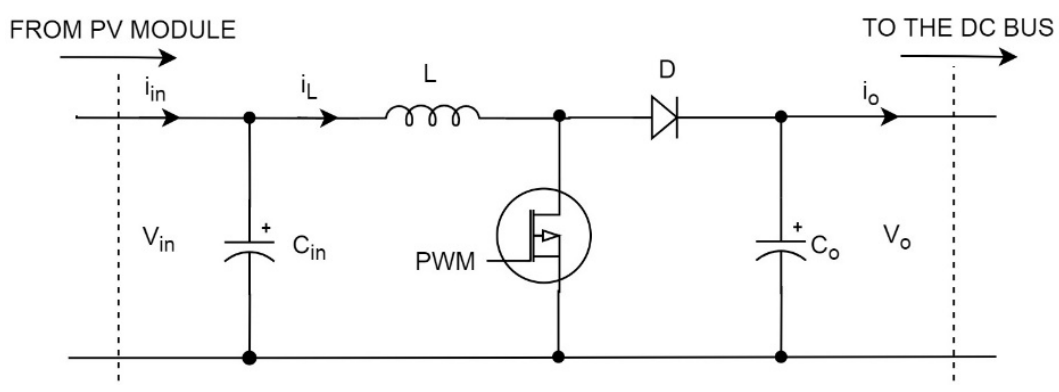

Figure 3. Boost DC/DC converter topology.

The boost converter is responsible for regulating the voltage from the PV modules by means of a controller, in this case, the adaptive Lyapunov controller, adjusting the on and off switching duration by means of pulse-width modulation (PWM). The operation of the converter depends on the state of the switch. When it is on, the diode is in reverse polarity, and the inductor stores energy, whereas the load is isolated. When the switch is off, the load receives the energy from the inductor. The boost converter can work in two different modes, namely, the continuous conduction mode (CCM) (when the inductor current is always higher than zero) and the discontinuous conduction mode (when the inductor current is zero for a time period). In PV systems, the operation mode of the $\mathrm{DC} / \mathrm{DC}$ converter is CCM.

The equations that model a boost converter are (1) and (2).

$$
\frac{d V_{i n}}{d t}=\frac{i_{i n}-i_{L}}{C_{i n}}
$$




$$
\frac{d i_{L}}{d t}=\frac{V_{i n}}{L}-V_{o} \gamma
$$

where $\gamma$ is the control signal that can be rewritten as $\gamma=\frac{1-D 1}{D 1}$ with $D_{1}$ as the duty cycle, $V_{i n}$ is the boost converter input voltage, $i_{i n}$ is its input current, $i_{L}$ is the inductor current, $C_{i n}$ is DC/DC converter input capacitor, and $V_{o}$ is the boost converter's output voltage.

In this study, the boost converter has been built to regulate the voltage from the PV system. The input voltage is $50 \mathrm{~V}$, whereas the input current is $4 \mathrm{~A}$. Thus, the maximum power transfer is $200 \mathrm{~W}$. The output voltage is $120 \mathrm{~V}$. The efficiency of the converter is $95 \%$, and its output voltage ripple is $0.8 \%$.

\subsubsection{Adaptive Lyapunov Control for the DC/DC Converter}

The adaptive Lyapunov control is designed to regulate the voltage at the PV modules' output and to reach the MPP under any environmental condition. This controller is applied to the DC/DC converter, in this case, the boost converter. Thus, the equations of the converter are required (Equations (1) and (2)). Then, an inner control loop is needed to manage the inductor current and an outer control loop for the DC/DC converter input voltage. Thus, the definition of the errors and the time derivative of the errors are in (3)-(6), where $e_{1}$ is the voltage error, and $e_{2}$ is the current error.

$$
\begin{gathered}
e_{1}=V_{i n}-V_{p v, r e f} \\
e_{2}=i_{L}-i_{L, r e f} \\
\dot{e}_{1}=\dot{V}_{i n}-\dot{V}_{p v, r e f} \\
\dot{e}_{2}=\dot{i}_{L}-\dot{i}_{i L, r e f}
\end{gathered}
$$

$V_{P V, \text { ref }}$ is the reference voltage that must be reached at the boost converter input, and $i_{L, \text { ref }}$ is the reference current that must be tracked by the inductor current.

The procedure now requires the definition of the Lyapunov function as $V_{1}=\frac{1}{2} e_{1}^{2}+\frac{1}{2} e_{2}^{2}$. Then, the time derivative of the Lyapunov function is presented in Equation (7).

$$
\dot{V}_{1}=e_{1} \underbrace{\left(\frac{i_{i n}-i_{L}}{C_{i n}}-\dot{V}_{p v, r e f}\right)}_{=-K_{1} e_{1}}+e_{2} \underbrace{\left(-\frac{1}{C_{i n}} e_{1}+\frac{1}{L} V_{i n}-\dot{i}_{L, r e f}\right)}_{=-K_{2} e_{2}}
$$

The time derivative of the Lyapunov function must be negative and for that, the two terms of Equation (7) must be equal to $-K_{1} e_{1}$ and $-K_{2} e_{2}$, with $K_{1}>0$ and $K_{2}>0$. Thus, the inductor current controller is achieved using Equation (8).

$$
i_{L, r e f}=i_{L}=i_{i n}-C_{i n} \dot{V}_{p v, r e f}+C_{i n} K_{1} e_{1}
$$

Differentiating Equation (8) with respect to time yields (9),

$$
\dot{i}_{i L, r e f}=\dot{i}_{i n}-C_{i n} \ddot{V}_{p v, r e f}-K_{1} e_{2}-C_{i n} K_{1}^{2} e_{1}
$$

and after substituting (9) in Equation (7), the expression becomes (10).

$$
-\frac{1}{C_{i n}} e_{1}+\frac{1}{L} V_{i n}-V_{o} \gamma-\dot{i}_{i n}+C_{i n} \ddot{V}_{p v, r e f}+K_{1} e_{2}+C_{i n} K_{1}^{2} e_{1}=-K_{2} e_{2}
$$

Then, the control law is (11).

$$
\gamma=\frac{1}{V_{o}}\left(-\frac{1}{C_{i n}} e_{1}+\frac{1}{L} V_{i n}-\dot{i}_{i n}+C_{i n} \ddot{V}_{p v, r e f}+\left(K_{1}+K_{2}\right) e_{2}+C_{i n} K_{1}^{2} e_{1}\right)
$$


To include the adaptive part of the parameters $C_{i n}$ and $L$, it is required to define the new parameters (12) and their errors (13), taking into consideration the estimated parameters $\hat{\theta}, \hat{\varnothing}, \hat{\omega}$. Thus, the controller does not depend on any physical parameter and adjusts itself automatically.

$$
\begin{gathered}
C_{\text {in }}=\theta \\
\frac{1}{L}=\varnothing \\
\frac{1}{C_{\text {in }}}=\omega \\
\widetilde{\theta}=\theta-\hat{\theta} \\
\widetilde{\varnothing}=\varnothing-\hat{\varnothing} \\
\widetilde{\omega}=\omega-\hat{\omega}
\end{gathered}
$$

Now it is necessary to update the control law to include the estimation of the adaptive parameters, $\widetilde{\theta}, \widetilde{\varnothing}, \widetilde{\omega}$, yielding (14).

$$
\gamma=\frac{1}{V_{o}}\left(-\hat{\omega} e_{1}+\hat{\varnothing} V_{i n}-\dot{i}_{i n}+\hat{\theta}\left(\ddot{V}_{p v, r e f}+K_{1}^{2} e_{1}\right)+\left(K_{1}+K_{2}\right) e_{2}\right)
$$

By including Equation (14) in (7), operating, and taking into account the adaptive parameters, the Lyapunov function becomes (15).

$$
\dot{V}_{1}=-K_{1} e_{1}^{2}-K_{2} e_{2}^{2}+e_{2}\left(-e_{1} \widetilde{\omega}-V_{i n} \widetilde{\varnothing}-\left(\ddot{V}_{p v, r e f}+K_{1}^{2} e_{1}\right) \widetilde{\theta}\right)
$$

Finally, the complete Lyapunov function is formulated in (19), taking into consideration all the errors, with $\{\rho, \sigma, \tau\} \in \Re^{+}$as constant adjustment parameters (16)-(18). For that, three new Lyapunov functions are required $-V_{2}, V_{3}$, and $V_{4}$. The adaptive Lyapunov function is $\mathrm{V}=V_{1}+V_{2}+V_{3}+V_{4}$.

$$
\begin{gathered}
\rho^{-1} V_{2}=\frac{1}{2} \rho^{-1} \theta_{1}^{2}=>\rho^{-1} \dot{V}_{3}=\rho^{-1} \tilde{\theta} \dot{\hat{\theta}} \\
\sigma^{-1} V_{3}=\frac{1}{2} \sigma^{-1} \varnothing_{1}^{2}=>\sigma^{-1} \dot{V}_{3}=\sigma^{-1} \widetilde{\varnothing} \dot{\hat{\varnothing}} \\
\tau^{-1} V_{4}=\frac{1}{2} \tau^{-1} \omega_{1}^{2}=>\tau^{-1} \dot{V}_{3}=\tau^{-1} \widetilde{\omega} \dot{\hat{\omega}} \\
\dot{V}=-K_{1} e_{1}^{2}-K_{2} e_{2}^{2}+\underbrace{\left(\tau^{-1} \dot{\hat{\omega}}-e_{1}\right)}_{=0} \widetilde{\omega}+\underbrace{\left(\sigma^{-1} \dot{\hat{\varnothing}}-V_{i n}\right)}_{=0} \widetilde{\varnothing}+\underbrace{\left(\rho^{-1} \dot{\hat{\theta}}-\ddot{V}_{p v, r e f}-K_{1}^{2} e_{1}\right)}_{=0} \widetilde{\theta}
\end{gathered}
$$

The obtained equations for the parameter adaptations are (20)-(22).

$$
\begin{aligned}
\tau^{-1} \dot{\hat{\omega}}-e_{1}=0 & \rightarrow \dot{\hat{\omega}}=\tau e_{1} \\
\sigma^{-1} \dot{\phi}-V_{i n}=0 & \rightarrow \dot{\varnothing}=\sigma V_{i n} \\
\rho^{-1} \dot{\hat{\theta}}-\ddot{V}_{p v, r e f}-K_{1}^{2} e_{1}=0 & \rightarrow \dot{\hat{\theta}}=\rho\left(\ddot{V}_{p v, r e f}+K_{1}^{2} e_{1}\right)
\end{aligned}
$$

The evolution of the parameters follows the dynamic obtained for Equations (20)-(22). Using these equations in the control law, the duty cycle of the DC/DC converter is achieved to reach the MPP taking into consideration the adaptation of the parameters such as the capacitor and the inductor.

Figure 4 summarizes the adaptive Lyapunov controller. It takes as input the reference voltage, $V_{P V}$,ref, which must be tracked and is given by the MPPT algorithm. The controller has two control loops. A faster inner control loop for the current and an outer control loop for the DC/DC converter input voltage. The error voltage, $e_{1}$, is calculated as the difference between the reference voltage $V_{P V}$,ref and the measured voltage at the boost converter input, $V_{i n}$. Then, using the voltage error and Equation (8), the reference current, 
$i_{L, \text { ref }}$ (called stabilization function) is achieved. This reference current is compared with the measured inductor current, $i_{L}$, obtaining the current error $e_{2}$. Thus, applying Equation (11) and integrating the parameters Equations (20)-(22), with respect to time, the duty cycle of the DC/DC converter, $\gamma$, is achieved.

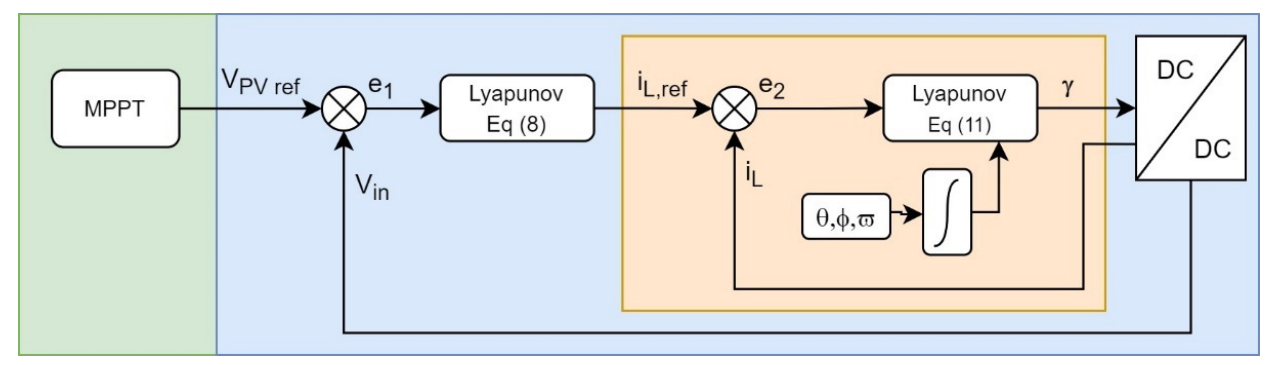

Figure 4. Flowchart of the adaptive Lyapunov control for the DC/DC converter.

Applying this controller, the duty cycle change rate is $7 \mathrm{~ms}$, the sampling time of the adaptive Lyapunov control is also $7 \mathrm{~ms}$, the PWM frequency is $25 \mathrm{kHz}$, and the resolution of the PWM is 10 bits.

\subsection{Wireless Conversion Unit (WCU)}

\subsubsection{DC/AC Converter}

The DC / AC converter, also known as an inverter, is the device responsible for converting the direct power (DC) from the PV system into alternating power (AC) for the electrical grid. Thus, the DC / AC converter connects the PV system to the electrical network. Moreover, the inverter has to provide the maximum active power to the grid.

In this study, a single-phase inverter is used. It is composed of four insulated-gate bipolar transistors (IGBT), IRGB4062DPB (International Rectifier, RS Components, Corby, UK), devices with anti-parallel diodes, as Figure 5 shows, driven by IR2106 devices. Those transistors are switched in a specific sequence to achieve the AC signals. The inverter output is connected to the grid by means of an output reactance, $L_{1}$, (with a value of $20 \mathrm{mH}$ ) to control the DC/AC converter output current $i_{0}$. Thus, the current injected into the grid, $i_{\text {grid }}$, has to be co-linear with the network voltage. Moreover, the current injected by the inverter must be nearly sinusoidal to avoid harmonic distortion. The boost converter output voltage is the inverter input voltage. This voltage must be controlled to keep it almost constant, which is achieved by means of a three-level PWM sinusoidal modulator.

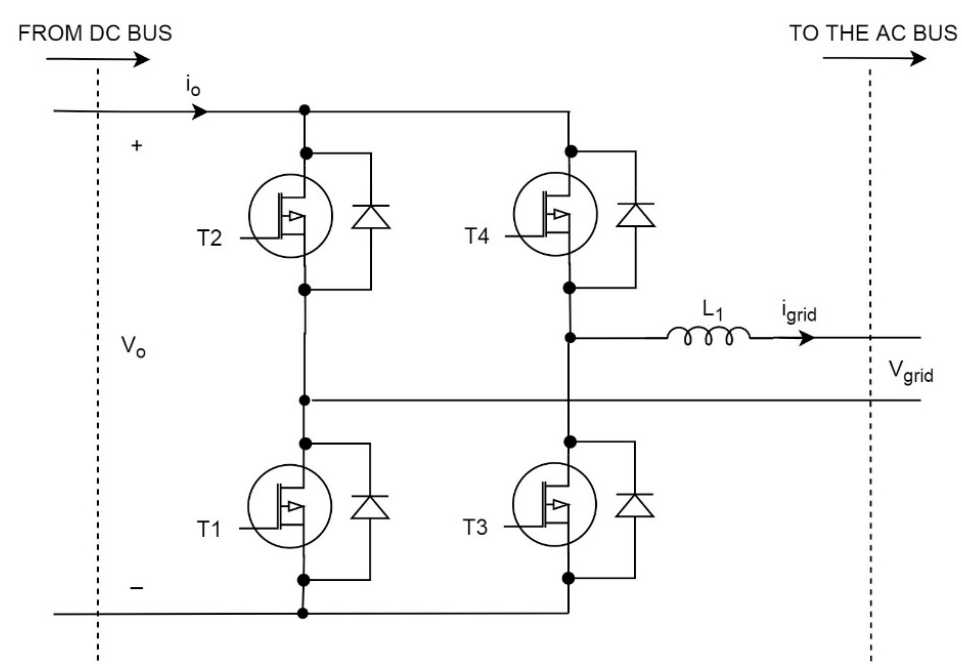

Figure 5. DC/AC converter topology. 
The DC/AC converter has been built in order to conduct the experiments. The maximum input voltage is $120 \mathrm{~V}$ and the maximum input current is $4.5 \mathrm{~A}$, whereas the maximum output voltage is $85 \mathrm{~V}$ and the maximum output power is $400 \mathrm{~W}$. The efficiency of the inverter is $94 \%$.

\subsubsection{DC/AC Converter Control}

The controller responsible for managing the inverter is a PI controller that generates a PWM signal to inject energy into the power grid. In this case, a cascade PI with an inner loop is used to control the inductor current. This inner loop is the fast loop, and it receives the sinusoidal reference current that the inverter should provide, $i_{\text {grid }(t), \text { ref }}$. In addition, the reference current $i_{\text {ref. }}$ is synchronized with the grid signal. This inner loop achieves the current error $e_{i}$. The outer control loop is responsible for controlling the DC/AC converter input voltage, $V_{o}$, and generating the sinusoidal reference current. In this loop, the input voltage is compared with the reference inverter input voltage, $V_{o, r e f}$, which must be reached, obtaining the voltage error $e_{V}$. The procedure is presented in Figure 6, where the duty cycle of the inverter, $\gamma_{i n v}$, is obtained. This control method has been used because it is effective and simple to implement, requiring a reduced computation time. The PWM frequency is $10 \mathrm{kHz}$ and the PWM resolution is 10 bits. The sampling time of the adaptive backstepping control loop is $0.1 \mathrm{~ms}$, whereas the duty cycle change rate is also $0.1 \mathrm{~ms}$.

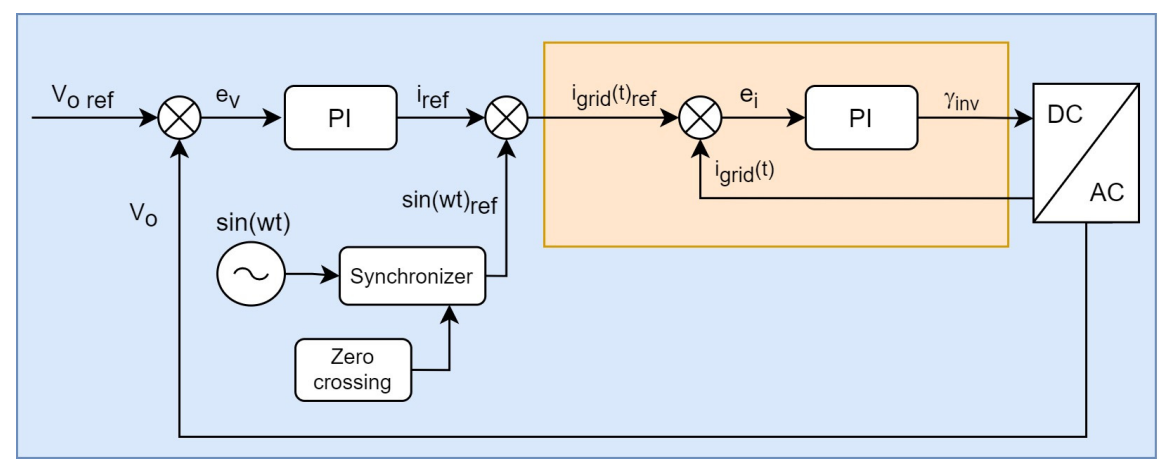

Figure 6. DC/AC converter's PI control.

\subsubsection{MPPT}

The WGPVS must search for the MPP to transfer the maximum power to the electrical network. For that, an MPPT algorithm is required. In this case, it includes a regression plane [26] in which all the MPPs are obtained for different values of temperature and irradiance (providing the theoretical reference voltage $V_{r e f, t}$ ) and a modified P\&O [26], which provides an incremental value of the reference voltage, $\Delta V_{\text {ref, }, p}$, where the MPP is located. Both the regression plane and the modified $\mathrm{P} \& \mathrm{O}$ are used to calculate the reference voltage to be reached by the adaptive Lyapunov controller, $V_{P V \text {,ref, }}$ to obtain the MPP. Figure 7 depicts the used MPPT algorithm.

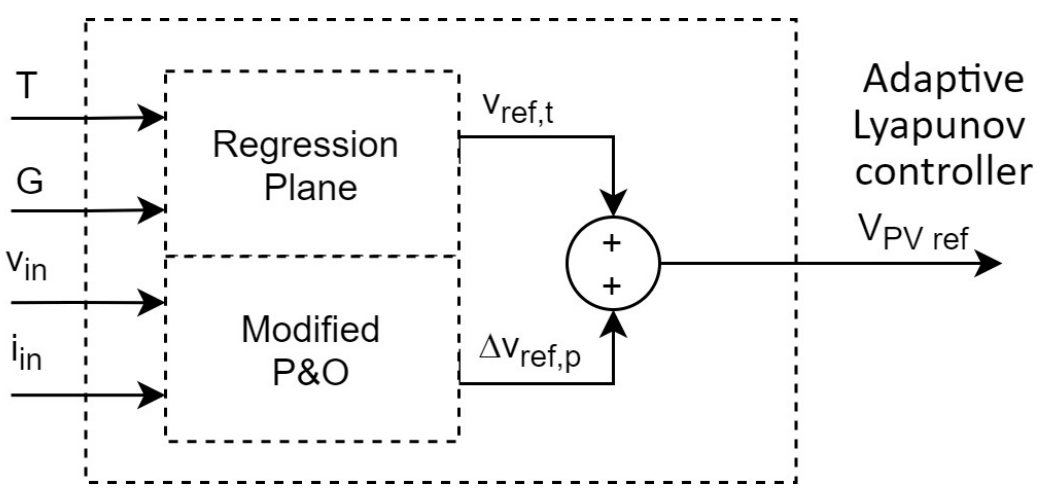

Figure 7. Block diagram of the maximum power point tracking (MPPT) algorithm. 


\section{Communication Module}

A WSN consists of nodes, which are sensor devices, and a coordinator or host, which is the controller of the network. The nodes send their data wirelessly to the coordinator. The choice of the technology to be used depends on the requirements of the target application, with considerations such as latency, security requirements, or battery life. Amongst the various WSN standards, such as the well-known IEEE 802.11 (Wi-Fi) and IEEE 802.15, the IEEE 802.15.4 standard shows high flexibility and is specifically designed for low power, low data rates, and low-cost sensor communication. For these networks, Wi-Fi is inappropriate because, even though it provides high data rates and superior range, its power consumption is an important disadvantage. Bluetooth low energy (BLE) is an interesting option for WSNs, but it has a short range. The IEEE 802.15.4 defined only the lowest two layers of the protocol stack, the physical layer (PHY) and the medium access control layer (MAC). The upper layers of the protocol stack are defined separately by other architectures, such as 6LoWPAN, ZigBee, ISA100.11a, and WirelessHART.

\subsection{Wireless Signal Flow}

The wireless, grid-connected PV system has a coordinator node, WCC, which is responsible for linking the communications and receiving the sensor data sent by the WSPS and the WCU nodes. The WCC receives the voltage, $V_{i n}$, the boost converter input current, $i_{i n}$, the inductor current, $i_{L}$, the temperature, $T$, and the irradiance, $G$, from the sensors of the WSPS every $7 \mathrm{~ms}$. Then, the WCC applies the adaptive Lyapunov control and generates the control signal, the duty cycle, $\gamma$, which is sent by the host to the WSPS. As for the data sent by the WCU, they are received every $0.1 \mathrm{~ms}$. The values sent by the WCU are the DC/AC converter input voltage, $V_{o}$, and the inverter output current, $i_{\text {grid }}$. With those values, the control signal, the duty cycle for the inverter, $\gamma_{i n v}$, is obtained after applying the cascade PI control in the WCC. This information is depicted in Figure 8, in which the communication between the host node and the two different controllers is presented. The host node sends the signal values to the controllers and the controllers calculate the duty cycle required for the correct operation of the system. Then, the control signals are sent by the host node to the WSPS and WCU. The two figures at the bottom of Figure 8 are the DC/DC controller and the DC/AC controller, showing the signals required to obtain the control signals. In the case of the DC/DC controller, the signals are the irradiance, the temperature, the boost converter input voltage and current, the DC/DC converter output voltage, and the inductor current. For the DC/AC controller, the signals needed are the grid current and the inverter input voltage, $V_{0}$.

The WSPS node is responsible for controlling the boost converter to extract the maximum power from the PV modules. To this purpose, the values of the DC/DC converter input voltage, the boost converter input current, the inductor current, the irradiance, and the temperature must be read by means of analog to digital converters (ADCs). Those values are sampled and sent to the host node every $7 \mathrm{~ms}$. Then, the WSPS node receives the control signal for the DC/DC converter.

The WCU node controls the DC/AC converter and is responsible for maintaining the inverter input voltage given by the reference value. For that, the sensor signals, in this case, the DC/DC converter output voltage and the DC/AC converter inductor current, $i_{\text {grid }}$, are sampled every $0.1 \mathrm{~ms}$. The received signal is the control signal for the inverter, $\gamma_{i n v}$, as Figure 9 presents. 


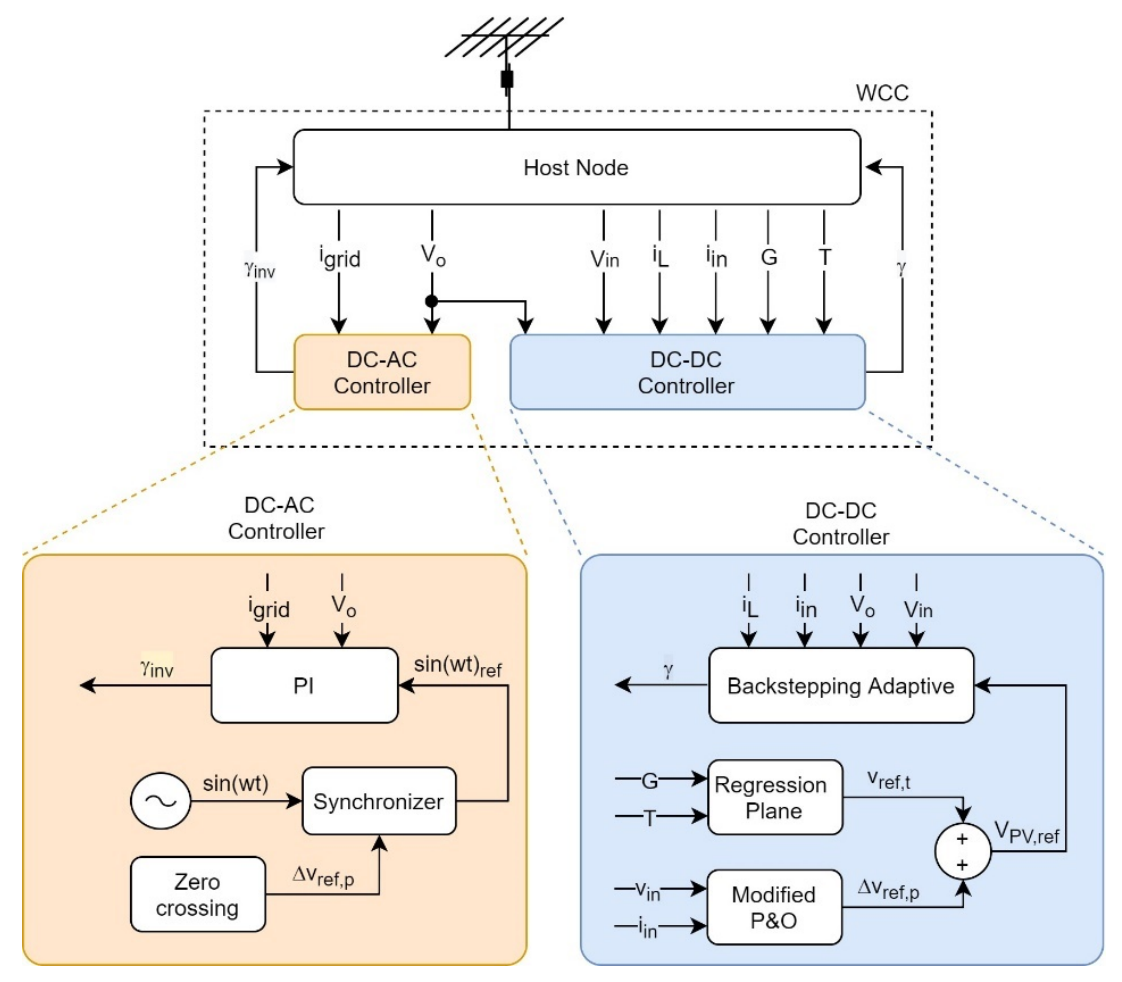

Figure 8. Wireless signal flow of the wireless centralized control (WCC) system.
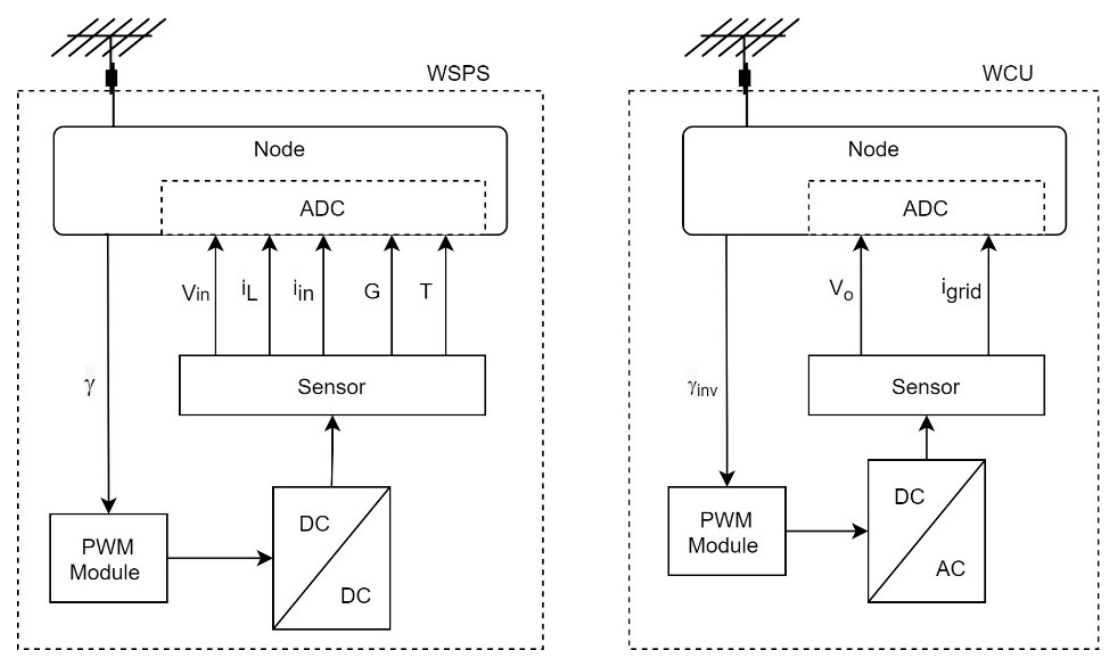

Figure 9. Wireless signal flow for the sensor nodes of the DC/DC converters and the inverters.

\subsection{Hardware of the Network Nodes}

Two sensor nodes send their data to the coordinator through wireless links. The coordinator uses the data locally. It is responsible for processing the data from the sensors and responding to every DC/DC converter and every inverter. As such, the coordinator manages the WSPS and the WCU as two relatively independent networks.

Despite their aforementioned advantages, proper attention must be paid when selecting the components of the WSNs because failure to do so could compromise the reliability of the monitoring system. The implementation of the network nodes is compact and features a versatile design, with a highly integrated and cost-effective solution. The proposed solution is power-aware, with care to the selection of the microprocessor, and power management is addressed in both the hardware and the software design. 
The hardware has been designed so that the network node can be configured as both a sensor node for the DC/DC converter and the inverter and a coordinator node. The SJ1 jumper allows this change of role of the node. Figure 10 shows the board of the wireless network node. It comprises a wireless module, sensors, a microcontroller, a differential analog to digital converter (ADC), and a power source. Several nodes have been deployed to configure the sensor network for the grid-connected PV system. Each unit is governed by an ATmega328 microcontroller from Atmel (San Jose, CA, USA) that processes information from the sensors. The set of sensors measure the required parameters, i.e., voltages, currents, temperature, and irradiance, and have analog outputs that are sampled by the external ADC. A second microcontroller ATmega128RFA1 (Atmel), which includes the wireless transceiver, controls the transmission stack of the IEEE 802.15.4 standard. These components are powered by a rechargeable $3.3 \mathrm{~V} / 2600 \mathrm{mAh}$ battery. The charge is maintained by the photovoltaic panel to achieve full energy autonomy when the network node performs as a sensor node. If it acts as a coordinator, it is powered from the base station. All components were selected to minimize the power consumption for the target application. The proposed dual-processor architecture allows balancing cost, power consumption, and performance. If a single microcontroller were used, it would be more difficult to achieve real-time monitoring and control of the PV system because the number of samples required for accurate tracking would be more limited.

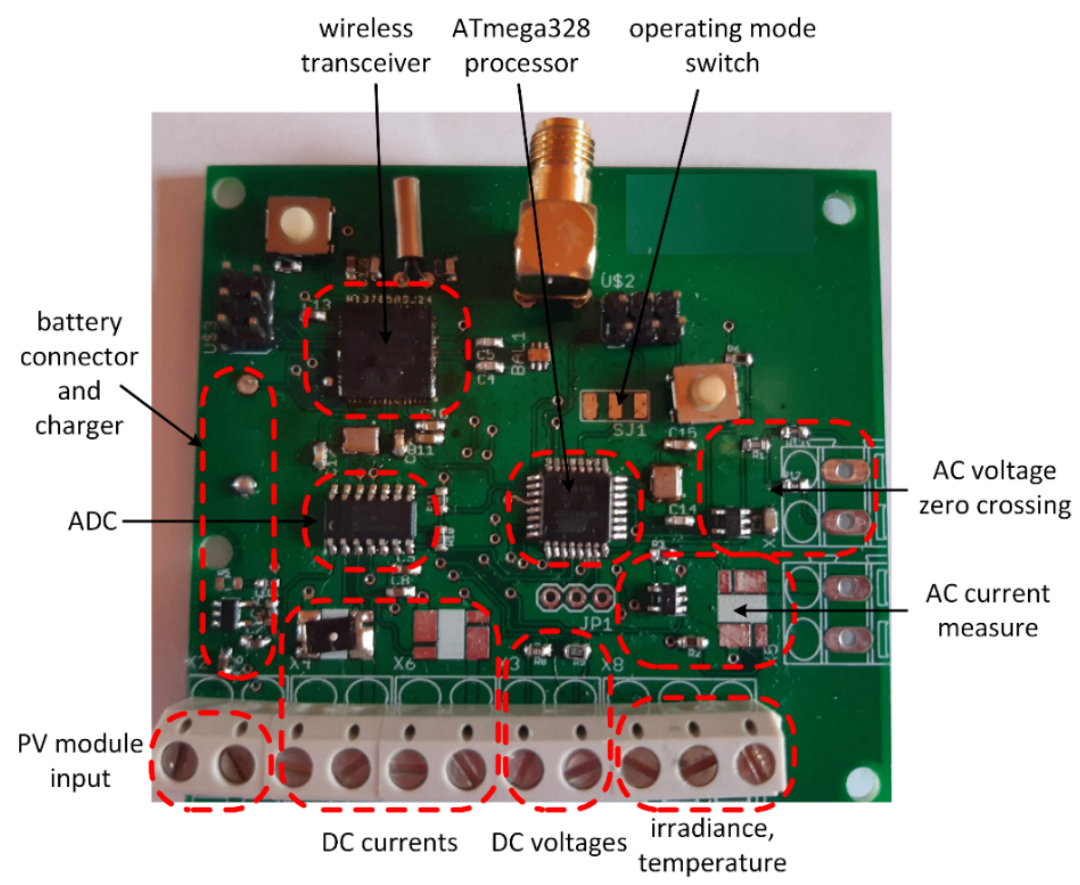

Figure 10. PCB of the network node.

(1) ATmega328 processor: An 8-bit microcontroller with 32 pins and Automatic Voltage Regulator (AVR) enhanced Reduced Instruction Set Computer (RISC) architecture, with an input voltage range from $1.8 \mathrm{~V}$ to $5.5 \mathrm{~V}$, and a $20 \mathrm{MHz}$ internal oscillator. It has up to 23 digital input/output pins, 8-channel 10-bit Analogue to Digital Converter (ADC), two Serial Peripheral Interface (SPI), one I2C and one Universal Synchronous/asynchronous Receiver Transmitter (USART) module, two 8-bit timers and one 16-bit timer, $32 \mathrm{~KB}$ of program memory, $1 \mathrm{~KB}$ of Electrically Erasable Programmable Read-Only Memory(EEPROM), 2 KB of Statis Random Access Memory (SRAM), and a real-time counter (RTC). The microcontroller is well suited for this application, given its low-power operating current, which is $200 \mu \mathrm{A}$ at $1.8 \mathrm{~V}$ and $1 \mathrm{MHz}$, and $0.75 \mu \mathrm{A}$ for standby current in power-save mode with $32 \mathrm{kHz}$ RTC.

(2) Single-chip wireless module: The short-range radio module is an ATmega128RFA1, which exchanges data via wireless communication at $2.4 \mathrm{GHz}$ following the IEEE 
802.15.4 standard. The ATmega128RFA1 is a low-power 8-bit microcontroller based on the AVR-enhanced RISC architecture combined with a high data rate transceiver. It handles the physical layer of the wireless communication, enables very robust transmission, and only uses a minimum number of external components. It combines excellent Radio Frequency (RF) performance with low cost, small size, and low current consumption. It has an input voltage range of $1.8 \mathrm{~V}$ to $3.6 \mathrm{~V}$ at $16 \mathrm{MHz}$ and has $128 \mathrm{~KB}$ of program memory, $4 \mathrm{~KB}$ of EEPROM, $16 \mathrm{~KB}$ of SRAM, and RTC. The supply current of the device is $14.5 \mathrm{~mA}$ at the maximum transmission output power of $3.5 \mathrm{dBm}$ and $12.5 \mathrm{~mA}$ in the reception state. The microcontroller and the transceiver provide various sleep modes, allowing the user to tailor the power consumption to the requirements of the application.

(3) External ADC: This device allows precise sensor data acquisition, while also improving software management and reducing the microcontroller load required for real-time monitoring. The MCP3428 (Microchip, Chandler, AZ, USA) is a 16-bit ADC with four-channel differential inputs. This configuration provides precision in the current measurement of up to $9 \mu \mathrm{A}$, which allows for the appropriate control of the DC/DC boost converter. Moreover, this ADC can be put in a low-power standby mode or shut down completely through simple pin-driven control, allowing improved power consumption control. The ADC data is read by the ATmega328 microcontroller through SPI.

(4) Sensors and sensor interface circuits: The measurements of current are carried out with resistors in the PCB, and voltage dividers for the required measurements of voltages. The temperature sensor uses four wires and the irradiance sensor uses two wires. All these analog values are periodically sampled under practical operation conditions by the differential ADC.

As it was shown in Figure 8, the DC/ AC controller needs a zero-crossing detector to synchronize the signal generated by the DC/AC converter with the grid. An integrator circuit using the micropower TLV2761IDBV (Texas Instruments, Dallas, TX, USA) operational amplifier has been used. Its output is directly connected to an external interrupt of the ATmega328 microcontroller. On the order hand, to measure the AC current of the grid, a four-pad current sensing resistor of $50 \mathrm{~m} \Omega$ from Vishay Precision Group (Malvern, PA, USA), with a maximum power of $3 \mathrm{~W}$, a tolerance of $0.1 \%$, a temperature coefficient of resistance of $15 \mathrm{ppm} /{ }^{\circ} \mathrm{C}$, and a maximum current of $54 \mathrm{~A}$, has been used. The voltage across the resistor is applied to a differential input amplifier circuit based on the same previously mentioned operational amplifier.

The coordinator node also acts as a gateway between the sensor network and the base station. The coordinator node is powered from an external source via USB and is connected directly to a PC via a Transistor-Transistor Logic to Universal Serial Bus (TTL-USB) transceiver, using the JP1 connector.

\subsection{Firmware}

The Atmel microcontrollers used in the network nodes follow a layered approach compatible with the IEEE 802.15.4 standard. The stack modules PAL, MAC (MAC-API), TAL, and Resources have been modified to the requirements of the grid-connected photovoltaic system. The beacon-enabled mode has been the mechanism chosen of the IEEE 802.15.4 standard to synchronize the bidirectional data transmission between the sensor nodes and the coordinator. The network coordinator is responsible for setting up the wireless network and follows the known negotiation protocol with the launching of beacons to receive the data sent by the sensor nodes, the association requests, and the confirmations by means of acknowledgment (ACK) frames. A slotted Carrier Sense Multiple Access with Collision Detection (CSMA/CA) is the mechanism that provides media access to the sensor nodes, synchronized by means of the beacons of the superframe structure. Nevertheless, sensor nodes also use GTS to avoid time delays in the data transmission and provide a predetermined data monitoring latency. This fact ensures that the controllers of both the 
boost converters and the inverters can operate stably and in real-time since they receive data at low latency intervals and within a specified time.

The coordinator (host) node configures the parameters required to create the network. Each sensor node gathers the PV module parameters and broadcasts an association beacon. Then, the coordinator associates a PV module number to the MAC address of each sensor node, and these can now forward the data to the coordinator in a reserved GTS. Next, the data is processed by the algorithms, generating the control signals for the DC/DC converters and the inverters, which return to each sensor node accordingly to track the MPPT and provide the energy from the PV modules to the grid in the correct way. The parameters Beacon Order $(\mathrm{BO})$ and Superframe Order $(\mathrm{SO})$ are defined in the standard and have been set to determine the time between beacons and the time in which the sensor node can transmit, respectively [27].

\section{Experimental Verification}

Figure 11 presents the experimental platform of the grid-connected PV system in which the experiments have been conducted. Part number 1 is the WCC, and number 2 consists of the boost converter and the sensors. Part number 3 includes the network nodes from the DC/DC converter and the DC/AC converter. Part number 4 is the inverter. Finally, part number 5 presents the connection of the DC/AC converter to the electrical network. As it can also be seen, a monitoring application is designed to visualize the sensor values and the control parameters to verify if the system is working properly. The sensors used to measure the currents and voltages are LEM sensors. For the voltage, LV25-P sensors are used whereas for the current the sensors are LA25-NP. The temperature is measured by an LM35 sensor and the irradiance is measured with a commercial irradiance meter.

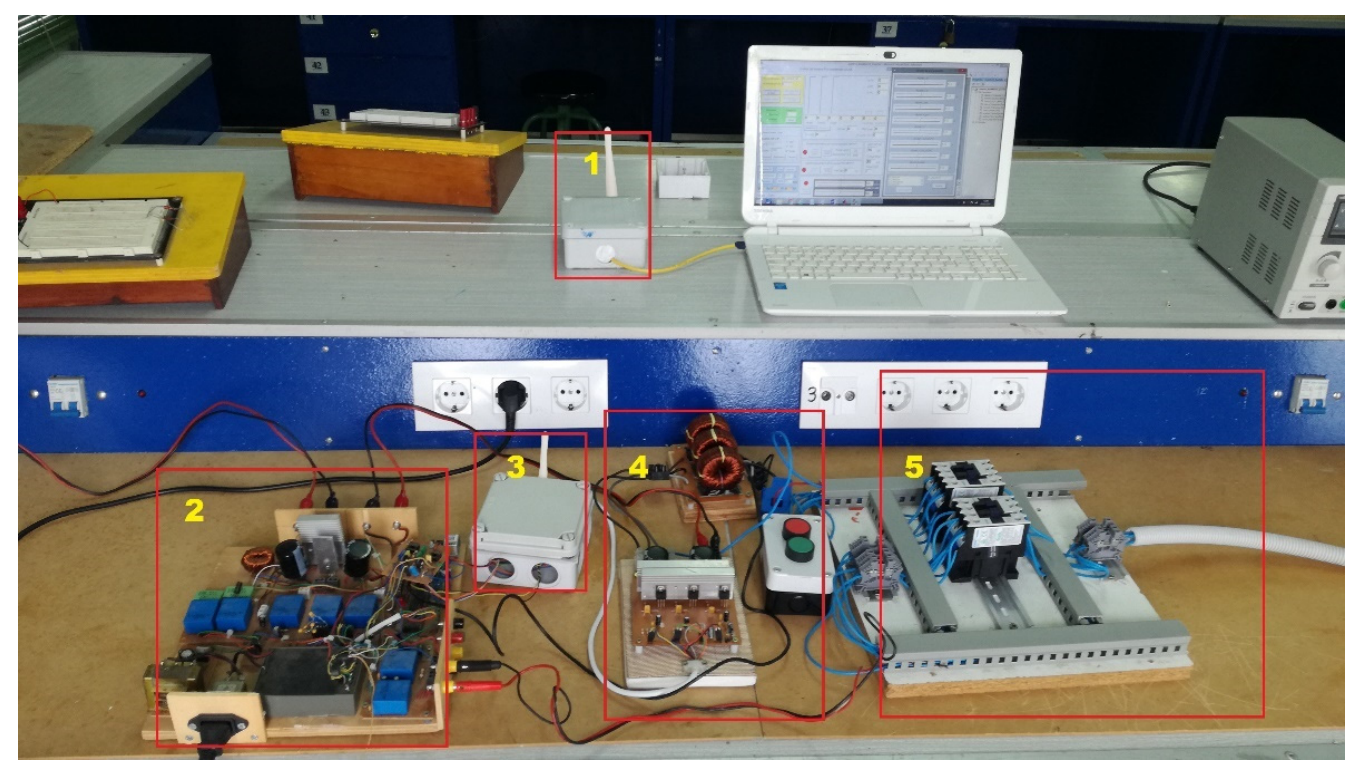

Figure 11. Experimental platform.

\subsection{Wireless Data Transmission Module}

Severe environments can cause temporary or permanent connection failure between sensor nodes. Successful data transmission from a sensor node to the coordinator relies on correct propagation between the nodes, with node failure potentially having a severe influence on network performance. To assess proper node operation, a diagnosis mechanism becomes necessary.

In order to test if the power generated by the converters can alter the wireless data transmission of the sensor nodes placed near them, experimental measurements have been performed to determine the quality of the signal (link quality indicator (LQI)). Figure 12 shows the measured results providing a signal loss of only $2 \mathrm{~dB}$ when the converter is 
stopped (blue line) compared to when it is put to its maximum power (red line), concluding the reliability of the data transmission under extreme working conditions of the converters.

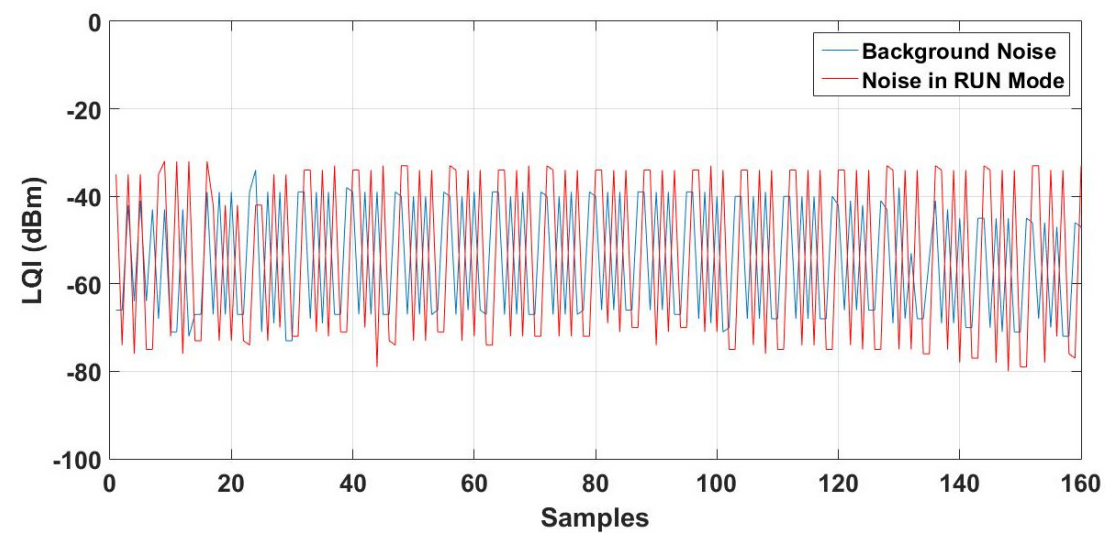

Figure 12. Quality of the signal under the influence of the converter.

Figure 13 shows the power consumption of the sensor node, measured in the laboratory. It is observed that it uses an average power of $14.55 \mathrm{~mW}$, while for the transmission of data it uses $46.77 \mathrm{~mW}$.

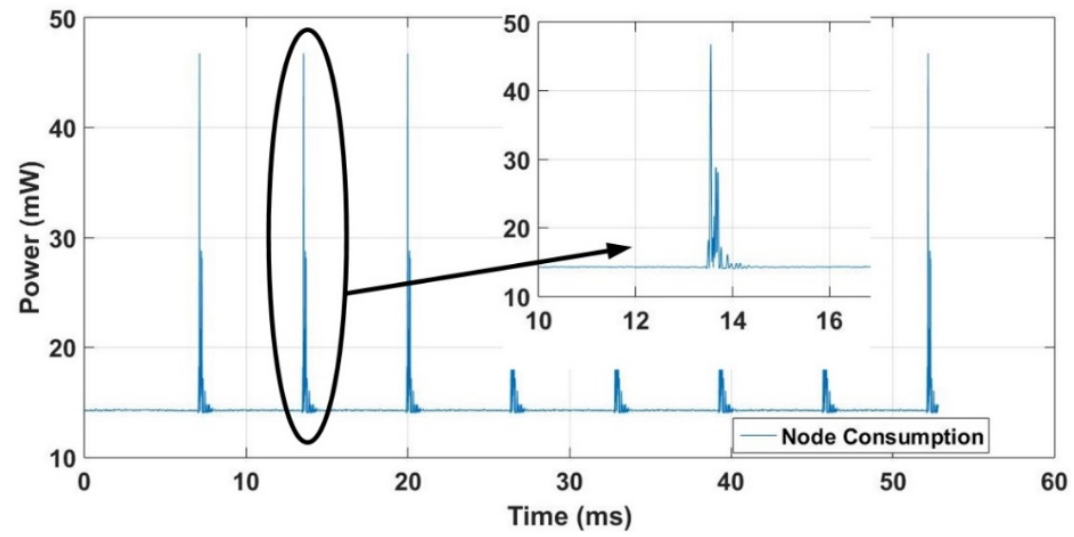

Figure 13. Measured power consumption of the network node.

\subsection{Experimental Results for the DC/DC Converter}

An experiment has been carried out for different levels of irradiance. Its value changes from $820 \mathrm{~W} / \mathrm{m}^{2}$ to $500 \mathrm{~W} / \mathrm{m}^{2}$ at $\mathrm{t}=7 \mathrm{~s}$, then to $835 \mathrm{~W} / \mathrm{m}^{2}$ at $\mathrm{t}=21 \mathrm{~s}$, and to $510 \mathrm{~W} / \mathrm{m}^{2}$ at $\mathrm{t}=29 \mathrm{~s}$. Finally, the irradiance takes a value of $838 \mathrm{~W} / \mathrm{m}^{2}$ at $38 \mathrm{~s}$. In this research, the changes have been considered step signals. With these irradiance variations, and consequently the change of the temperature, the operation of the wireless grid-connected PV system is tested. Table 2 summarizes the time intervals and the obtained values of irradiance, temperature, and reference voltage that provide the MPP, and the MPP.

Table 2. Values at the maximum power point (MPP).

\begin{tabular}{ccccc}
\hline $\begin{array}{c}\text { Time } \\
(\mathbf{s})\end{array}$ & $\begin{array}{c}\text { Irradiance } \\
\left(\mathbf{W} / \mathbf{m}^{\mathbf{2}}\right)\end{array}$ & $\begin{array}{c}\text { Temperature } \\
\left({ }^{\circ} \mathbf{C}\right)\end{array}$ & $\begin{array}{c}\mathbf{V}_{\mathbf{P V}, \text { ref }} \\
(\mathbf{V})\end{array}$ & $\begin{array}{c}\text { PMPP } \\
(\mathbf{W})\end{array}$ \\
\hline$[0-7)$ & 820 & 38.1 & 17.5 & 51.1 \\
{$[7-21)$} & 500 & 38.3 & 18 & 30.1 \\
{$[21-29)$} & 835 & 37.9 & 17.4 & 52.2 \\
{$[29-38)$} & 510 & 38.1 & 17.9 & 31.4 \\
{$[38-55]$} & 838 & 37.9 & 17.5 & 52.5 \\
\hline
\end{tabular}


Figure 14 shows the voltages at the DC/DC converter input and output. Moreover, it presents the reference voltage that must be tracked to reach the MPP. The boost converter output voltage reaches around $54 \mathrm{~V}$ when the irradiance is high, whereas the voltage takes a value of about $53 \mathrm{~V}$ when the irradiance is low. The input voltage is between $17.4 \mathrm{~V}$ and $18 \mathrm{~V}$, depending on the time interval where the signal is located. As it is shown, the voltage signal is properly tracked, obtaining an MPPT tracking efficiency between $99.05 \%$ and $99.95 \%$.

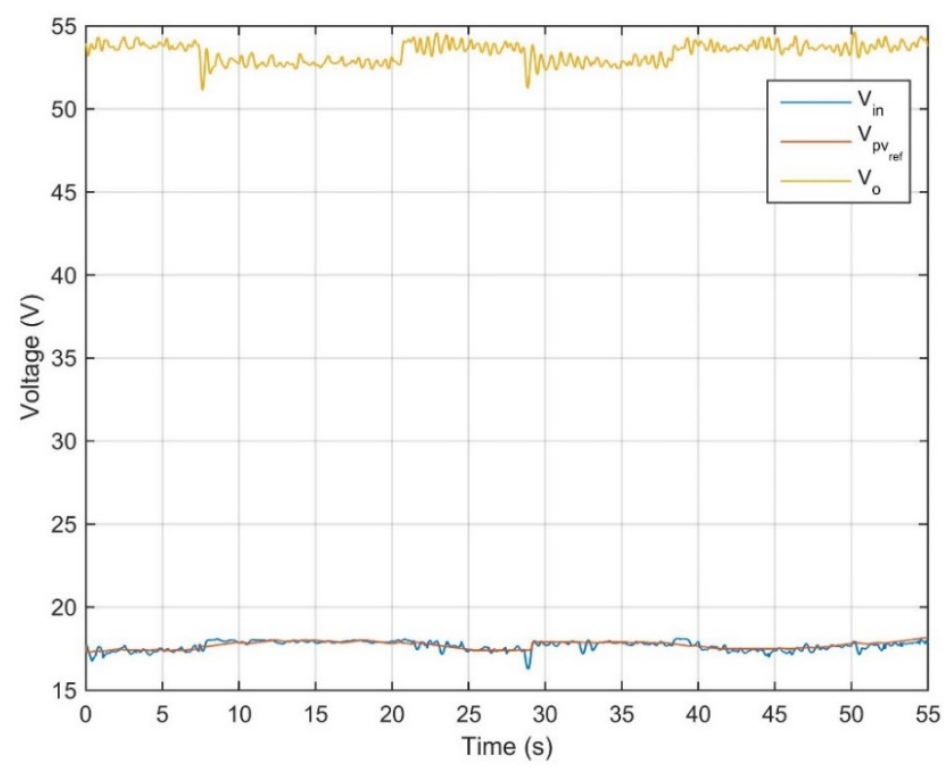

Figure 14. DC/DC converter voltage profiles: reference voltage and DC/DC converter input and output voltage.

Figure 15 depicts the inductor current and the DC/DC converter input and output current. The same as the output voltage signals, the values of the current signals increase when the irradiance is high, whereas their values drop when the irradiance is low.

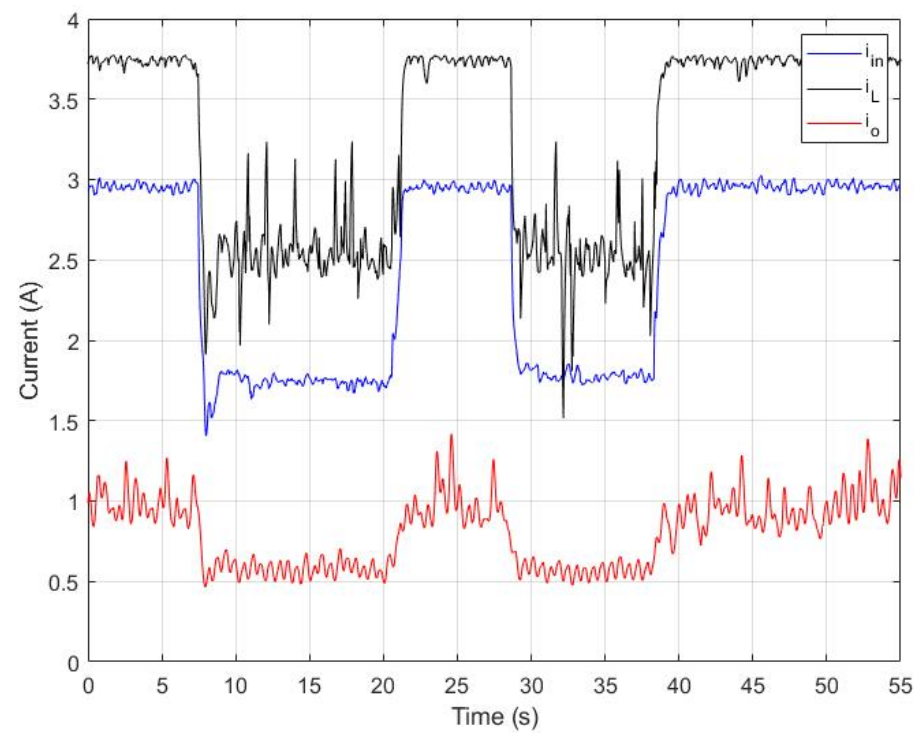

Figure 15. DC/DC converter current profiles: inductor current and DC/DC converter input and output current.

Figure 16 presents the parameter estimation of the capacitor and inductor of the boost converter. It shows how the parameters are readjusted by the controller in each change 
to stabilize the system. The system is robust under uncertainty of the parameters and environmental conditions.

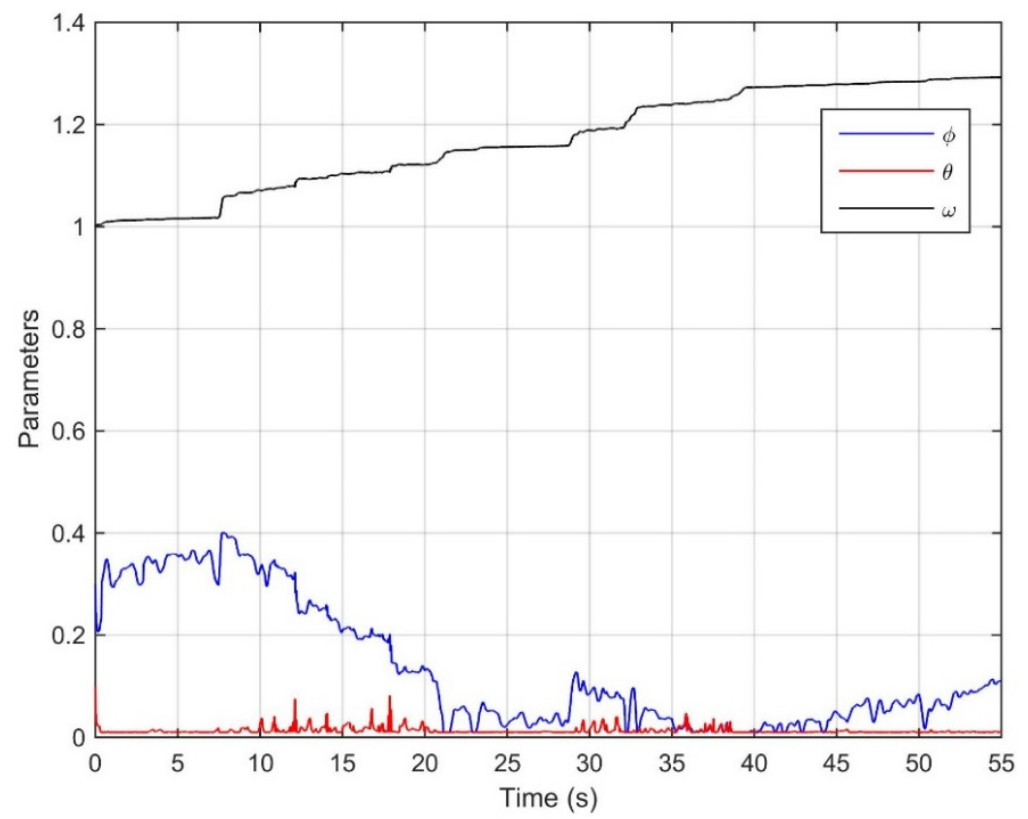

Figure 16. Evolution of the adaptive parameters.

The power at the DC/DC converter input and output are presented in Figure 17. The input power reaches a value of approximately $52 \mathrm{~W}$ for the high irradiance and about $31 \mathrm{~W}$ for the interval with the low irradiance. The output power takes slightly lower values than the input power due to the commutation losses in the boost converter. Thus, the efficiency of the boost converter is defined as the relationship between the output power and the input power, achieving a value that ranges from $93 \%$ to $98 \%$. Moreover, the MPPT efficiency is also achieved by taking into consideration the values of the input power and the real MPP, reaching an efficiency higher than $99.05 \%$.

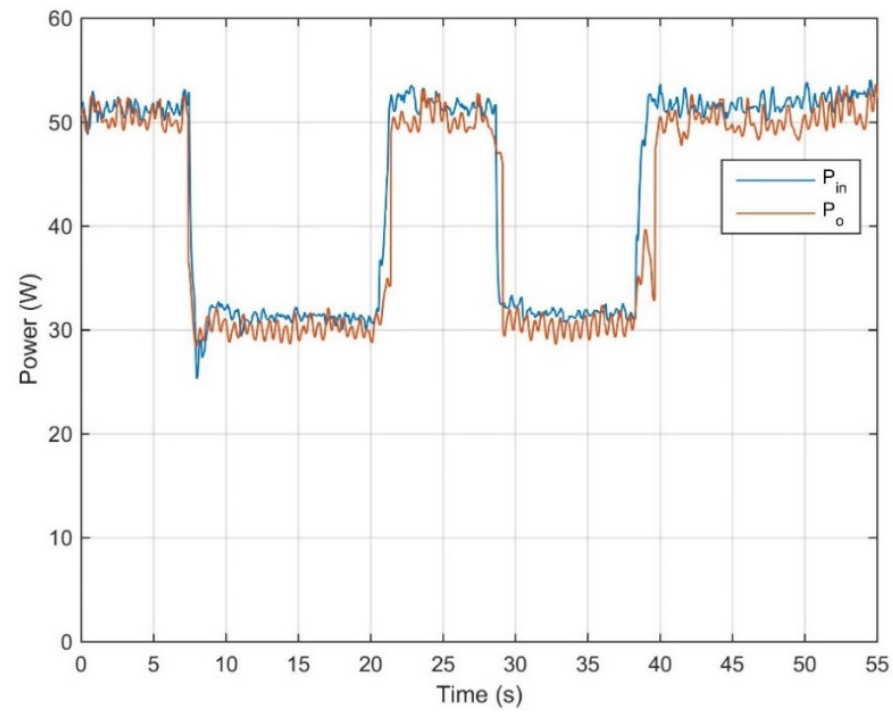

Figure 17. DC/DC converter power evolution.

Figure 18 shows the control signal using adaptive Lyapunov control in the boost converter. The value of the control signal varies when there is a perturbation, i.e., when the irradiance changes. The duty cycle takes a value between $77.9 \%$ and $78.6 \%$ when the 
irradiance is high, and it decreases to between $76 \%$ and $76.5 \%$, approximately, when the irradiance drops.

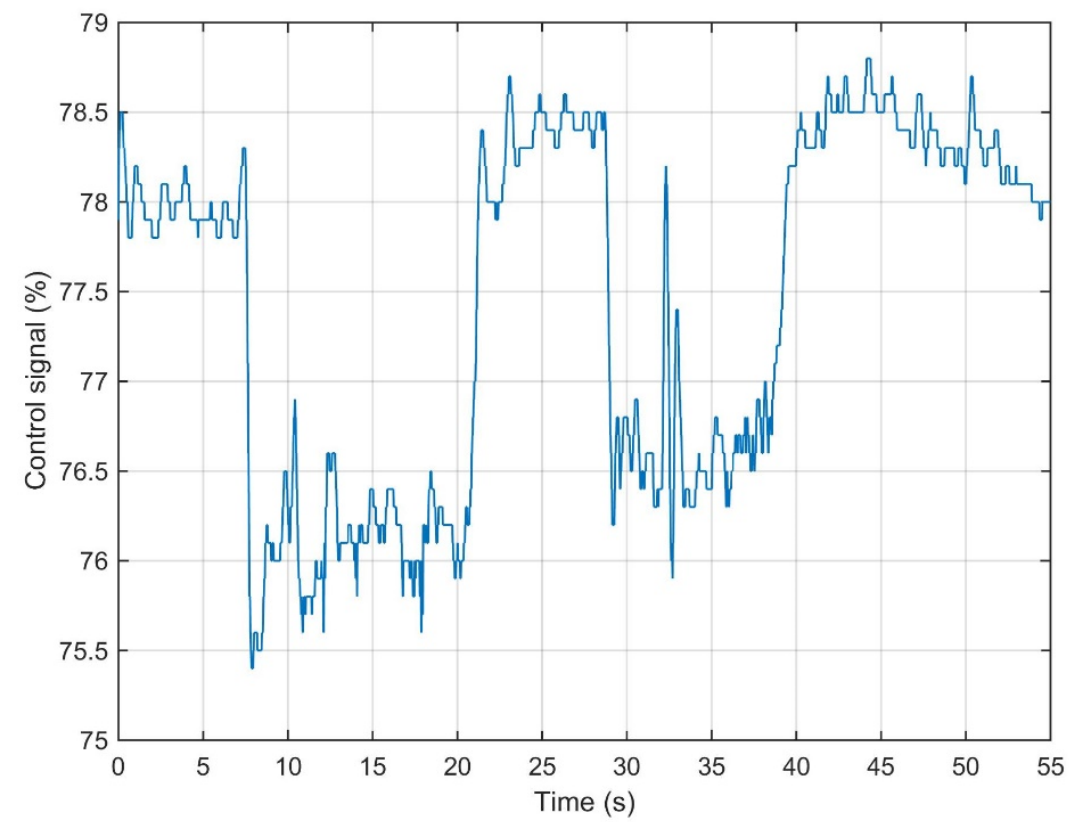

Figure 18. DC/DC converter control signal.

The results conclude that the operation of the wireless grid-connected PV system is robust when there are sudden variations in the environmental conditions. The reference voltage is tracked and the maximum power is reached in all the conditions. Thus, the robustness of the adaptive Lyapunov controller is also verified even under external perturbations.

\subsection{Experimental Results for the DC/AC Converter}

In this section, the results for the $\mathrm{DC} / \mathrm{AC}$ converter are shown, taking into consideration the results obtained for the $\mathrm{DC} / \mathrm{AC}$ converter since the output values for the boost converter are the input values for the inverter. In this case, the first two intervals are considered and the results are shown. Then, four signals are presented to validate the operation of the DC/AC converter. The signals are the DC/DC converter input voltage, $V_{i n}$, the boost converter input current, $i_{i n}$, the DC/AC converter input voltage, $V_{o}$, and the grid current, $i_{\text {grid }}$. For $820 \mathrm{~W} / \mathrm{m}^{2}$, the voltage at the inverter input, $V_{o}$, is $53.8 \mathrm{~V}$, and the current provided by the DC/DC converter is $0.87 \mathrm{~A}$, with transferring power of $46.8 \mathrm{~W}$. The $\mathrm{DC} / \mathrm{DC}$ converter transfers power of $51.01 \mathrm{~W}$, then the inverter efficiency is $92 \%$. For an irradiance of $500 \mathrm{~W} / \mathrm{m}^{2}$, the voltage at the inverter input is $52.7 \mathrm{~V}$ and the current is $0.6 \mathrm{~A}$, whereas the power provided by the DC/DC converter is $31.62 \mathrm{~W}$ and the power transferred to the power grid is $29.9 \mathrm{~W}$, obtaining, in this case, an efficiency of $94.5 \%$. Table 3 presents the values for the inverter input current, the DC/AC converter input and output voltage, and the inverter output current.

Table 3. Values of DC/DC converter input voltage and current, DC/AC converter input voltage, and grid current for different irradiances, i.e., $820 \mathrm{~W} / \mathrm{m}^{2}$ for the first interval and $500 \mathrm{~W} / \mathrm{m}^{2}$ for the second.

\begin{tabular}{ccccccccc}
\hline $\begin{array}{c}\text { Time } \\
(\mathbf{s})\end{array}$ & \multicolumn{2}{c}{$\mathbf{i}_{\text {in }}$} & \multicolumn{2}{c}{$\mathbf{V}_{\text {in }}$} & \multicolumn{2}{c}{$\mathbf{V}_{\mathbf{O}}$} & \multicolumn{2}{c}{$\mathbf{i}_{\text {igrid }}$} \\
$(\mathbf{p u})$ & $\mathbf{( A )}$ & $\mathbf{( p u )}$ & $\mathbf{( V )}$ & $\mathbf{( p u )}$ & $\mathbf{( V )}$ & $\mathbf{( p u )}$ & $\mathbf{( A )}$ \\
\hline$[0-0.2)$ & 0.87 & 2.93 & 0.62 & 17.41 & 0.98 & 53.8 & 1 & 1.19 \\
{$[0.2-0.45]$} & 0.49 & 1.66 & 0.64 & 18.01 & 0.96 & 52.7 & 0.69 & 0.74 \\
\hline
\end{tabular}


Figure 19 shows the DC/DC converter input voltage, $V_{i n}$, and current, $i_{i n}$, the inverter input voltage, $V_{0}$, and the inverter output current as a sinusoidal waveform. When the irradiance changes, there is a change in the parameter values, with the DC/DC converter output voltage and current dropping, while $V_{\text {in }}$ increases its value to reach the MPP. Moreover, the inverter output current decreases its value with the irradiance. The inverter injects low harmonic distortion, with a total harmonic distortion (THD) of $2 \%$.

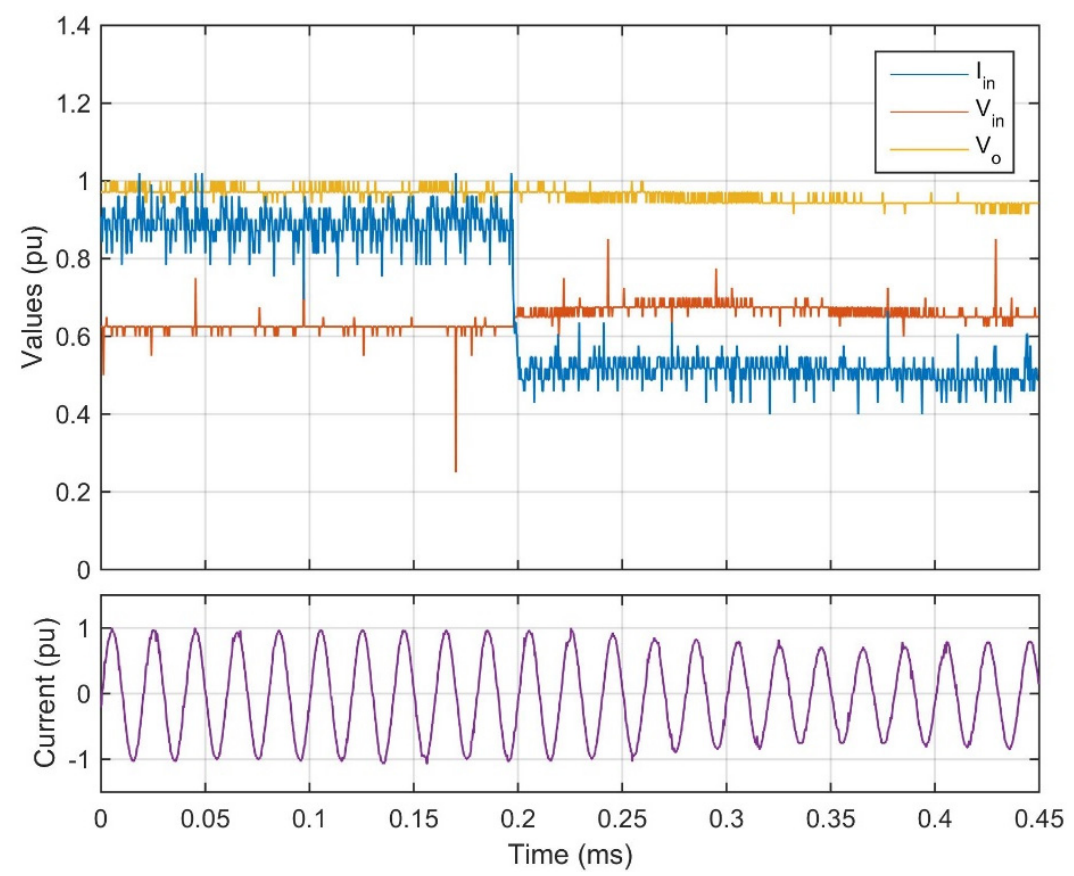

Figure 19. PV system signals: boost converter input voltage and current and DC/AC converter input voltage and output current.

\section{Conclusions}

A great number of monitoring applications are based on WSNs, given their advantages in terms of costs by drastically reducing the required cabling, their scalability, flexibility, and variety of network topologies, in addition to the ease of deployment and reduced maintenance. WSNs have successfully provided monitoring solutions in many fields, including PV power plants. Nowadays, many efforts are devoted to increasing the energy production of PV plants, which can be monitored to control the proper operation of the system and assess its production. This paper presents a wireless solution for a gridconnected PV system with two approaches for the control of the DC/DC converters and the inverters. The communication module has been developed to achieve the required low latency for the controllers to operate stably and in real-time. Communications are built using the IEEE 802.15.4 protocol because the aim is to construct a WSN with low power consumption, low complexity, and low data transmission rate. A low-cost wireless multi-sensor node has been deployed with a versatile design, which is able to perform as both a sensor node and a coordinator node. Long-term operation is allowed using solar cells and rechargeable batteries solution to power the nodes, requiring minimum human intervention.

Experimental results show an MPPT efficiency higher than $99.05 \%$ by applying an adaptive Lyapunov control for the boost converter, which transfers energy with an efficiency between $93 \%$ and $98 \%$, and for the DC/AC converter, the power transferred to the power grid ranges from $92 \%$ to $94.5 \%$, injecting nearly sinusoidal grid currents at near unity power factor since the THD is only $2 \%$. The robustness of the wireless grid-connected PV system has also been verified even under external perturbations in the environmental conditions, ensuring the stability of the system even when there are sudden changes in the irradiance. 
Author Contributions: Conceptualization, A.D.M. and J.A.G.-G.; methodology, A.D.M. and J.A.G.-G.; software, J.M.-G. and J.M.C.; validation, J.M.-G. and J.M.C.; formal analysis, A.D.M. and J.A.G.-G.; investigation, J.M.-G., A.D.M., J.M.C., J.A.G.-G. and A.H.; resources, J.M.-G. and J.M.C.; data curation, J.M.-G., J.M.C. and A.H.; writing-original draft preparation, A.D.M. and J.A.G.-G.; writing-review and editing, A.H.; visualization, J.M.-G.; supervision, A.D.M.; project administration, J.M.C. and J.A.G.-G. All authors have read and agreed to the published version of the manuscript.

Funding: This research received no external funding.

Conflicts of Interest: The authors declare no conflict of interest.

\section{References}

1. Hariri, M.; Mat Desa, M.K.; Masri, S.; Atiqi Mohd Zainuri, M.A. Grid-Connected PV Generation System-Components and Challenges: A Review. Energies 2020, 13, 4279. [CrossRef]

2. Kim, K.A.; Seo, G.S.; Cho, B.H.; Krein, P.T. Photovoltaic Hot-Spot Detection for Solar Panel Substrings Using AC Parameter Characterization. IEEE Trans. Power Electron. 2016, 31, 1121-1130. [CrossRef]

3. Shazly, A.M.; Montaser, A.E.S. A comparative study of $\mathrm{P} \& \mathrm{O}$ and INC maximum power point tracking techniques for gridconnected PV systems. SN Appl. Sci. 2019, 1, 174.

4. Barth, C.; Pilawa-Podgurski, R.C.N. Dithering Digital Ripple Correlation Control for Photovoltaic Maximum Power Point Tracking. IEEE Trans. Power Electron. 2015, 30, 4548-4559. [CrossRef]

5. Fang, Y.; Zhu, Y.; Fei, J. Adaptive Intelligent Sliding Mode Control of a Photovoltaic Grid-Connected Inverter. Appl. Sci. 2018, 8, 1756. [CrossRef]

6. Rezk, H.; Aly, M.; Al-Dhaifallah, M.; Shovama, M. Design and Hardware Implementation of New Adaptive Fuzzy Logic-Based MPPT Control Method for Photovoltaic Applications. IEEE Access 2019, 8, 106427-106438. [CrossRef]

7. Zecevic, Z.; Rolevski, M. Neural Network Approach to MPPT Control and Irradiance Estimation. Appl. Sci. 2020, 10, 5051. [CrossRef]

8. Hu, K.; Cao, S.; Li, W.; Zhu, F. An Improved Particle Swarm Optimization Algorithm Suitable for Photovoltaic Power Tracking Under Partial Shading Conditions. IEEE Access 2019, 7, 143217-143232. [CrossRef]

9. Verma, D.; Nema, S.; Shandilya, A.M.; Dash, S.K. Maximum power point tracking (MPPT) techniques: Recapitulation in solar photovoltaic systems. Renew. Sustain. Energy Rev. 2016, 54, 1018-1034. [CrossRef]

10. Martin, A.D.; Cano, J.M.; Silva, J.F.A.; Vazquez, J.R. Backstepping Control of Smart Grid-Connected Distributed Photovoltaic Power Supplies for Telecom Equipment. IEEE Trans. Energy Convers. 2015, 30, 1496-1504. [CrossRef]

11. Gupta, A.; Thakur, R.; Murarka, S. An Efficient Approach to Zero Crossing Detection Based on Opto-Coupler. Int. J. Eng. Res. Appl. 2013, 3, 834-838.

12. Wang, X.; Pi, Y.; Mao, W.; Huang, H. Network Coordinated Power Point Tracking for Grid-Connected Photovoltaic Systems. IEEE J. Select. Areas Commun. 2014, 32, 1425-1440. [CrossRef]

13. Paredes-Parra, J.M.; Mateo-Aroca, A.; Silvente-Niñirola, G.; Bueso, M.C.; Molina-García, A. PV Module Monitoring System Based on Low-Cost Solutions. Wirel. Raspberry Appl. Assess. Energies 2018, 11, 3051.

14. Bonfiglio, A.; Brignone, M.; Delfino, F.; Procopio, R. Optimal Control and Operation of Grid-Connected Photovoltaic Production Units for Voltage Support in Medium-Voltage Networks. IEEE Trans. Sustain. Energy 2014, 5, 254-263. [CrossRef]

15. Weckx, S.; D’hulst, R.; Driesen, J. Locational Pricing to Mitigate Voltage Problems Caused by High PV Penetration. Energies 2015, 8, 4607-4628. [CrossRef]

16. Sreekanth, T.; Lakshminarasamma, N.; Mishra, M.K. A Single-Stage Grid-Connected High Gain Buck-Boost Inverter with Maximum Power Point Tracking. IEEE Trans. Energy Convers. 2017, 32, 330-339. [CrossRef]

17. Mohammed, S.A.Q.; Rafaq, M.S.; Choi, H.H.; Jung, J.W. A Robust Adaptive PI Voltage Controller to Eliminate Impact of Disturbances and Distorted Model Parameters for 3-Phase CVCF Inverters. IEEE Trans. Ind. Inform. 2020, 16, 2168-2176. [CrossRef]

18. Hwang, M.H.; Kim, Y.G.; Lee, H.S.; Kim, Y.D.; Cha, H.R. A Study on the Improvement of Efficiency by Detection Solar Module Faults in Deteriorated Photovoltaic Power Plants. Appl. Sci. 2021, 11, 727. [CrossRef]

19. Martínez-Martínez, J.; Aponte-Roa, D.; Vergara-Laurens, I.; Weaver, W.W. A Low-Cost Secure IoT Mechanism for Monitoring and Controlling Polygeneration Microgrids. Appl. Sci. 2020, 10, 8354. [CrossRef]

20. Ando, B.; Baglio, S.; Pistorio, A.; Tina, G.M.; Ventura, C. Sentinella: Smart Monitoring of Photovoltaic Systems at Panel Level. IEEE Trans. Instrum. Meas. 2015, 64, 2188-2199. [CrossRef]

21. Shariff, F.; Rahim, N.A.; Ping, H.W. Zigbee-based data acquisition system for online monitoring of grid-connected photovoltaic system. Expert Syst. Appl. 2015, 42, 1730-1742. [CrossRef]

22. Le, P.T.; Tsai, H.L.; Lam, T.H. A wireless visualization monitoring, evaluation system for commercial photovoltaic modules solely in MATLAB/Simulink environment. Solar Energy 2016, 140, 1-11. [CrossRef]

23. Moon, S.; Kim, S.J.; Seo, J.W.; Park, J.H.; Park, C.; Chung, C.S. Maximum power point tracking without current sensor for photovoltaic module integrated converter using Zigbee wireless network. Electr. Power Energy Syst. 2014, 56, 286-297. [CrossRef]

24. Moreno-Garcia, I.M.; Palacios-Garcia, E.J.; Pallares-Lopez, V.; Santiago, I.; Gonzalez-Redondo, M.J.; Varo-Martinez, M.; Real-Calvo, R.J. Real-Time Monitoring System for a Utility-Scale Photovoltaic Power Plant. Sensors 2016, 16, 770. [CrossRef] 
25. Llaria, A.; Terrasson, G.; Curea, O.; Jiménez, J. Application of Wireless Sensor and Actuator Networks to Achieve Intelligent Microgrids: A Promising Approach towards a Global Smart Grid Deployment. Appl. Sci. 2016, 6, 61. [CrossRef]

26. Vazquez, J.R.; Martin, A.D. Backstepping Control of a Buck-Boost Converter in an Experimental PV-System. J. Power Electron. 2015, 15, 1584-1592. [CrossRef]

27. Khan, S.; Alvi, A.N.; Khan, M.Z.; Awais Javed, M.; Alhazmi, O.H.; Bouk, S.H. A novel superframe structure and optimal time slot allocation algorithm for IEEE 802.15.4-based Internet of things. Int. J. Distrib. Sens. Netw. 2020, 16, 35692. [CrossRef] 